Original

\title{
Riesgo de cáncer de mama en trabajadoras de turno nocturno
}

\section{Breast cancer risk in night female workers}

\section{Francisco Javier Fresneda Moreno' José Carlos Gómez Santana² Hugo Bascopé Quintanilla'}

1. Unidad Docente de Medicina del Trabajo Madrid 1. Escuela Nacional de Medicina del Trabajo. Instituto de Salud Carlos III. Madrid. España.

2. Unidad Docente de Medicina del Trabajo de Castilla La Mancha.

Recibido: 27-12-12

Aceptado: 26-02-13

\section{Correspondencia}

Francisco Javier Fresneda Moreno

Hospital Universitario "Ramón y Cajal"

Ctra. Colmenar Viejo, kn. 9, 100

28034 Madrid. España

TIf: 646134471

Correo electrónico: j.fresneda@hotmail.com

Resumen

Los estudios epidemiológicos más recientes a nivel mundial han puesto de manifiesto una asociación entre el trabajo nocturno (15-20\% de los trabajadores) y un aumento del riesgo de cáncer de mama, siendo esta, una patología de gran prevalencia y la primera causa de neoplasia en el sexo femenino.

En el año 2007, la IARC consideró el trabajo nocturno como probable cancerígeno (categoría 2A).

Los turnos nocturnos producen una disrupción del ritmo circadiano. La exposición a la luz artificial nocturna inhibe la síntesis de melatonina aumentando el estímulo estrogénico, e inhibiendo el efecto antimitótico, antiangiogénico y antioxidante de esta hormona, aumentando así el riesgo tumoral.

Los objetivos son: identificar la evidencia científica existente entre la asociación trabajo nocturno y cáncer de mama, los factores intrínsecos/extrínsecos que pueden modificar la asociación causal, conocer desde el punto de vista científico la sostenibilidad de la melatonina en la chrono-disrupción y los factores de vulnerabilidad.

Se ha realizado la búsqueda bibliográfica de artículos publicados entre 2006-diciembre 2012 (exceptuando un meta-análisis del 2005), en las siguientes bases datos: Medline, Wok, Lilacs, OSH, IBECS, Cochrane, Scopus y Embase.

Se seleccionaron por nivel de evidencia y criterios de inclusión-exclusión un total de 15 artículos.

Se sugiere la existencia de una asociación no concluyente entre el riesgo de cáncer de mama y trabajo nocturno, por lo que se deben seguir realizando estudios más precisos en diferentes tipos de profesiones, etnias, minimizando la posibilidad de sesgos.

Med Segur Trab (Internet) 2013; 59 (230) 146-158

Palabras clave: Cáncer de mama, trabajo a turnos, turno nocturno, melatonina. 


\section{Abstract}

Recent epidemiological studies worldwide have shown an association between night shift (15-20\% of workers) and an increased risk of breast cancer, this being a highly prevalent disease and the leading cause of neoplasia in women.

In 2007, the IARC considered night shift as a probable carcinogen (category 2A).

Night shifts produce a circadian rhythm disruption. The exposure to artificial light at night produce an inhibition in the synthesis of melatonin, increasing the estrogenic stimulus, and inhibiting the antimitotic, antiangiogenic and antioxidant effects of this hormone, increasing the risk of neoplasia.

The aims of this review are to identify the scientific evidence in the association between night shift and breast cancer, to identify the intrinsic or extrinsic factors that can modify this causal association and to improve the knowledge concerning the scientific sustainability of the effect of melatonin in the chronodisruption and vulnerabilities for breast cancer in woman.

A literature search has been performed from 2006 to December 2012 from the following scientific databases: Medline, Wok, Lilacs, OSH, IBECS, Cochrane, Scopus and Embase.

15 articles were selected taking into account the level of evidence, according with SGIN criteria, and the full-filed inclusion and exclusion criteria.

The results suggests there are not a conclusive association between risk for breast cancer and night shift. So further studies should be more accurate in different occupations, ethnic groups, minimizing the possibility of bias.

Med Segur Trab (Internet) 2013; 59 (230) 146-158

Key words: Breast cancer, shift work, night shift, melatonin. 


\section{INTRODUCCIÓN}

En el año 1996 se publicó un estudio en Noruega que mostró una posible asociación entre el cáncer de mama y el trabajo por turnos, en una cohorte de operadoras de radio y telégrafo entre 1920-1980 ${ }^{29}$. Desde entonces se han realizado estudios a nivel mundial en relación con el riesgo de cáncer de mama y turnos de trabajo, más específicamente turnos nocturnos.

El trabajo en turnos de noche es frecuente en la actualidad; se estima que alrededor de un $15-20 \%$ de los trabajadores realiza su labor a turnos que incluyen trabajos nocturnos ${ }^{1}$.

El cáncer de mama es el tumor maligno más frecuente en el sexo femenino. Su incidencia es variable a nivel mundial. Estas diferencias se deben a los diversos hábitos de vida, los factores ambientales, los sistemas de notificación de cada país y a los procedimientos diagnósticos empleados. Su incidencia aumenta con el nivel socioeconómico, diagnosticándose más de la mitad del total de casos en los países desarrollados ${ }^{2}$.

Según datos de la Agencia Internacional de Investigación del Cáncer (IARC), la tasa de incidencia estandarizada estimada para España fue de 93,6 casos por 100.000 mujeresaño en 2006, frente a los 110 casos por cada 100.000 mujeres en Europa ${ }^{2 ; 4}$.

En España se diagnostican unos 22.000 casos al año, según datos de la Asociación Española contra el Cáncer de junio de 2011, lo que representa casi el $30 \%$ de todos los tumores del sexo femenino. La mayoría de los casos se diagnostican entre los 45 y 65 años (es decir, en edad laboral) y se estima que el riesgo de padecer cáncer de mama es aproximadamente 1 de cada 8 mujeres ${ }^{2 ; 5}$.

La etiología del cáncer de mama es multifactorial interviniendo factores de riesgo tanto laborales como no laborales.

En relación con el cáncer ocupacional y la incorporación progresiva de la mujer en el mundo laboral, se han descrito nuevos riesgos específicos del sexo femenino relacionados con su actividad laboral ${ }^{6}$.

En los últimos años se han identificado sustancias y factores que incrementan el riesgo de desarrollar cáncer de mama en mujeres trabajadoras de sectores concretos, como la exposición a agentes solventes, algunos pesticidas, metales, el trabajo nocturno y el tabaco ${ }^{2}$.

Dentro de los factores de riesgo laboral en el cáncer de mama se distinguen los riesgos asociados a sustancias químicas, a radiaciones y otro tipo de riesgos, entre los que destacan el trabajo a turnos y en especial el trabajo nocturno.

En 2007, la International Agency for Research on Cancer (IARC), consideró el trabajo nocturno como probable cancerígeno (Categoría 2A). Esta conclusión se basó en suficiente evidencia de estudios en animales de experimentación y en evidencia limitada en humanos.

En relación a lo anterior, cabe como posible hipótesis el hecho de que la exposición a la luz artificial nocturna produzca una disrupción del ritmo circadiano y como consecuencia una disminución en la síntesis de la hormona melatonina que juega un papel importante en la carcinogénesis ${ }^{1}$.

Muchas funciones fisiológicas, tales como la temperatura, la secreción hormonal, la alternancia sueño-vigilia y el estado de alerta se incrementan y decrecen en ciclos de aproximadamente 24 horas (ritmo circadiano). Un grupo de células en el cerebro, el Núcleo supraquiasmatico (NSQ), actúa como un marcapasos o reloj endógeno, dirigiendo los ritmos circadianos. Los factores ambientales, llamados sincronizadores o Zeitgbers ayudan a regular los ritmos circadianos en ciclos de 24 horas (día-noche). De estos factores (temperatura externa, presión atmosférica, etc.) la luz es el más poderoso sincronizador ${ }^{7}$. 
La melatonina (N-Acetil-5-metoxitriptamina) es una hormona principalmente secretada durante la noche por la glándula pineal. El nivel pico máximo de secreción de melatonina ocurre durante el sueño a mitad de la noche (entre las 00:00 h y las 03.00 h). Los factores que modulan su secreción son de 2 tipos: ambientales (fotoperiodo, temperatura, estación del año) y endógenos (la edad, a partir de los 30 años se produce una disminución de su síntesis en los humanos; y el estrés) Se ha demostrado que la melatonina está involucrada en la regulación de los ritmos circadianos, del sueño, envejecimiento, enfermedades cardiovasculares, afecciones psiquiátricas como el Desorden Afectivo Estacional (SAD). Se le atribuye una acción antineoplásica a través de los siguientes mecanismos: antioxidante, antimitótico y antiangiogénico. Puede actuar como un agente modulador del sistema inmunológico y alterar el metabolismo lipídico. Interviene en la regulación del crecimiento tumoral actuando a través de genes supresores de tumor y a nivel del cáncer mamario actúa bloqueando los receptores estrogénicos ER alfa e inhibiendo la aromatasa disminuyendo el estímulo estrogénico ${ }^{7 ; 28}$.

El trabajo en turnos de noche es frecuente en la actualidad. Se estima que alrededor de un $15-20 \%$ de los trabajadores realiza su labor a turnos que incluyen trabajos nocturnos ${ }^{1}$. El trabajo nocturno, bien fijo o más habitualmente a turnos, afecta más frecuentemente a los trabajadores de las ramas de actividades sanitarias y sociales $(23,8 \%)$ y química, saneamiento y extractiva (22,5\%). Las mujeres trabajan más frecuentemente en jornadas continuas de mañana y continuas de tarde, en cambio los hombres en turnos rotativos que incluyen la noche ${ }^{8}$.

La realización de turnos de trabajo es mucho más frecuente entre los trabajadores más jóvenes, especialmente los menores de 25 años. Por su parte, la frecuencia con que se trabaja en horario nocturno (al menos tres horas entre las 22:00 y las 3:00) varía significativamente entre hombre y mujer ${ }^{8}$.

El Estatuto de los Trabajadores (art. 36.1, párrafo 1) define los conceptos de trabajo y trabajador nocturno según el Estatuto de los Trabajadores en los siguientes términos: "Se considera trabajo nocturno al realizado entre las diez de la noche y las seis de la mañana".

Hemos de señalar que este concepto es más protector que el expresado en la Directiva 93/104 que recoge el período de las $00 \mathrm{~h}$ a las 5 de la mañana.

Ese mismo artículo define al trabajador nocturno en los siguientes términos: "Se considera trabajador nocturno al que realice normalmente en período nocturno una parte no inferior a tres horas de su jornada diaria de trabajo, así como aquel que se prevea que puede realizar en tal periodo una parte no inferior a un tercio de su jornada de trabajo anual".

\section{OBJETIVOS}

La presente revisión sistemática tiene como objetivo principal el de identificar la evidencia científica de la asociación existente entre trabajo nocturno y cáncer de mama.

Formulándose los siguientes objetivos secundarios:

- Identificar los factores intrínsecos/ extrínsecos que pueden modificar la asociación causal entre trabajo nocturno y cáncer de mama.

- Corroborar la sostenibilidad, desde el punto de vista de la evidencia científica, la teoría de la "Chrono-disruption" en la síntesis de la melatonina como causa principal del incremento del riesgo de cáncer de mama.

- Conocer los factores de vulnerabilidad para cáncer de mama en las mujeres trabajadoras de turnos nocturnos. 


\section{MATERIAL Y MÉTODOS}

Se realizó una búsqueda bibliográfica de artículos científicos publicados entre 2006 y 2013 (última fecha consultada 15/01/2013), incluyéndose un meta-análisis publicado en 2005.

Las bases de datos consultadas fueron: MEDLINE, WOK, LILACS, OSH, COCHRANE, SCOPUS, IBECS y EMBASE, utilizándose los descriptores y ecuaciones de búsqueda que figuran en la tabla 1.

Tabla 1. Bases de datos y ecuación de búsqueda empleados

\begin{tabular}{|c|c|}
\hline Bases de datos & Descriptores y ecuación de búsqueda \\
\hline MEDLINE & $\begin{array}{l}\left.1^{\circ}\right) \text { "breast neoplasms" [MeSH Terms] AND "work schedule Tolerance "[MeSH Terms]. } \\
2^{\circ} \text { ) "breast neoplasms" [MeSH Terms] AND "shift work" [MeSH Terms]. } \\
3^{\circ} \text { ) "breast cancer" [MeSH Terms] AND "night work" [MeSH Terms]. } \\
4^{\circ} \text { ) "breast neoplasms" [MeSH Terms] AND "night shift work" [MeSH Terms]. } \\
\text { Filters: Humans. From } 2006 \text { to } 2012 .\end{array}$ \\
\hline WOK & $\begin{array}{l}\left.1^{\circ}\right) \text { "breast neoplasms" AND "shiftwork". } \\
2^{\circ} \text { ) "breast neoplasms"AND "work Schedule Tolerance". }\end{array}$ \\
\hline LILACS & $\left.1^{\circ}\right)$ "cancer mama" [Descriptor asunto] and "trabajo turno" [Descriptor asunto]. \\
\hline OSH UPDATE & $\begin{array}{l}\left.1^{\circ}\right) \text { "breast neoplasms" AND "shift work". } \\
2^{\circ} \text { ) "breast neoplasms" AND "work Schedule Tolerance". }\end{array}$ \\
\hline IBECS & $1^{\circ}$ ) "cancer mama" AND "trabajo turno". \\
\hline COCHRANE & $\begin{array}{l}1^{\circ} \text { ) " breast neoplasms" [MeSH descriptor] + "shift work" [MeSH descriptor]. } \\
2^{\circ} \text { ) "breast neoplasms" [MeSH descriptor]+ "work Schedule Tolerance" [MeSH descriptor]. }\end{array}$ \\
\hline SCOPUS & $\begin{array}{l}\left.1^{\circ}\right) \text { "breast neoplasms" AND "shift work". } \\
\left.2^{\circ}\right) \text { "breast neoplasms" AND "work Schedule Tolerance". }\end{array}$ \\
\hline EMBASE & $\begin{array}{l}\left.1^{\circ}\right) \text { "breast neoplasms" AND "shift work". } \\
2^{\circ} \text { ) "breast neoplasms" AND "work Schedule Tolerance". }\end{array}$ \\
\hline
\end{tabular}

En base a los criterios de inclusión y exclusión (tabla 2,3) se seleccionan los artículos recuperados para su posterior lectura sistemática.

Tabla 2. Criterios de inclusión

\begin{tabular}{ll}
\hline \multicolumn{1}{c}{ Variables } & \multicolumn{1}{c}{ Criterios de inclusión } \\
\hline Artículos seleccionados & Los que relacionan el trabajo nocturno y el riesgo de cáncer de mama. \\
\hline Tamaño de la muestra & $\mathrm{n}>150$. \\
\hline Tipo de diseño & Estudios observacionales analíticos. \\
\hline Población & Mujeres trabajadoras. \\
\hline Artículos publicados & Fecha $>2006$. \\
\hline
\end{tabular}

Tabla 3. Criterios de exclusión.

\begin{tabular}{ll}
\hline \multicolumn{1}{c}{ Variables } & \multicolumn{1}{c}{ Criterios de exclusión } \\
\hline Criterios de inclusión & El no cumplir alguno de estos criterios. \\
\hline Duplicados & En distintas bases de datos / autor. \\
\hline Estudios no originales & \\
\hline
\end{tabular}

Una vez seleccionados los títulos y resúmenes de los artículos, se evaluaron de forma independiente por cada investigador, dirimiéndose las controversias mediante revisión conjunta y consenso sobre la pertinencia de su inclusión en la lectura sistemática, siguiendo los criterios descritos anteriormente.

Posteriormente se procedió a la recuperación de artículos, a texto completo, a través de la biblioteca de la Escuela Nacional de Medicina del Trabajo y la biblioteca del Hospital Universitario Ramón y Cajal (Madrid). 
Para la lectura sistemática se diseñó una tabla de captura de la información incluyendo los aspectos relacionados con: la identificación de artículo, características metodológicas y de la población y resultados y conclusiones (tabla 4).

Tabla 4. Variables estudiadas

\begin{tabular}{lll}
\hline \multicolumn{1}{c}{ Identificación } & \multicolumn{1}{c}{ Método } & \multicolumn{1}{c}{ Resultados y conclusiones } \\
\hline Título & Diseño & Resultados (HRs, RR, OR, IC 95\%) \\
\hline Autor/es & Tamaño muestral & Nivel de evidencia \\
\hline Año de publicación & Objetivos & \\
\hline Lugar estudio & Variables de exposición & \\
\hline Población (etnia, edad y actividad) & Variables de efecto & \\
\hline & Control de sesgos & \\
\hline
\end{tabular}

Para determinar la evidencia científica de cada artículo, se evaluó con los criterios SIGN de la "Scottish Intercollegiate Guidelines Network".

\section{RESULTADOS}

La tabla 5 refleja el número total de artículos recuperados en cada una de las bases de datos consultadas tras aplicar las distintas ecuaciones de búsqueda así como el número de artículos seleccionados para su revisión sistemática de acuerdo al proceso de selección seguido (figura 1).

Tabla 5. Número de artículos recuperados y seleccionados

\begin{tabular}{lcc}
\hline \multicolumn{1}{c}{ Bases de datos } & Artículos recuperados & Artículos seleccionados \\
\hline MEDLINE & 117 & 15 \\
\hline WOK & 0 & 0 \\
\hline LILACS & 0 & 0 \\
\hline IBECS & 0 & 0 \\
\hline OSH UPDATE & 0 & 0 \\
\hline COCHRANE & 0 & 0 \\
\hline SCOPUS & 10 & 0 \\
\hline EMBASE & 2 & 0 \\
\hline Total de artículos & 129 & 15 \\
\hline
\end{tabular}

Figura 1. Resultados de la búsqueda.

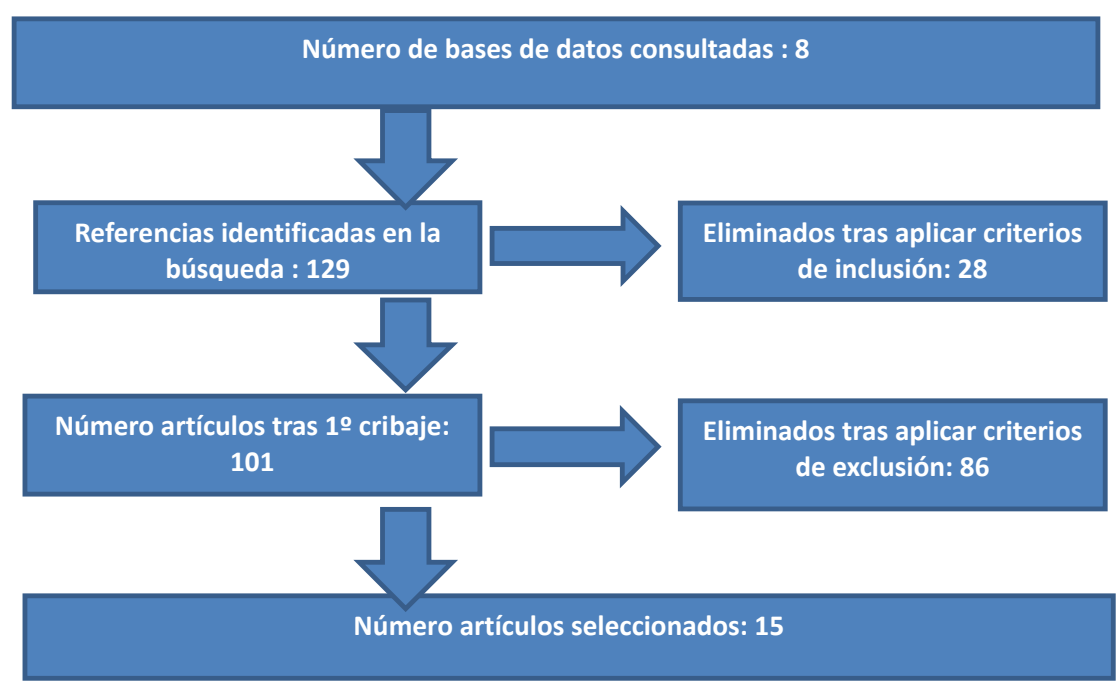


Se recuperaron un total de 129 referencias; de las cuales 28 fueron eliminadas por no cumplir los criterios de inclusión. De las 101 referencias restantes, válidas para la revisión, se procedió a la lectura de los resúmenes, eliminando 86 artículos, por cumplir alguno de los criterios de exclusión. El número total de artículos seleccionados para su lectura sistemática fueron de 15 .

Desde el punto de vista del análisis metodológico el tipo de diseño de los estudios incluidos en la revisión fueron: 1 meta-análisis, 5 estudios de cohortes y 9 estudios de casos-control.

Medgal et al ${ }^{10}$ (2005), realiza un meta-análisis de 13 estudios observacionales sobre riesgo de cáncer de mama ( 7 en mujeres de tripulación de cabina aérea y 6 en mujeres en turno nocturno). Estimando una OR global de 1,48, (IC 95\%: 1,36-1,61). Para riesgo de cáncer de mama en tripulación femenina de cabina obtiene una riesgo de incidencia estandarizada de 1,44, (IC 95\%: 1,26-1,65). Para mujeres en otros trabajados nocturnos obtiene un Riesgo Relativo de 1,51, (IC 95\%: 1,36-1,68). Evidenciando un aumento de riesgo en estas poblaciones de trabajadoras para el cáncer de mama.

Schernhammer Es ${ }^{11}$ (2006), estudia una cohorte prospectiva de 115.022 enfermeras pre-menopáusicas, evidenciando un aumento de riesgo de cáncer de mama en enfermeras con más de 20 años de trabajo nocturno rotatorio, en relación con aquellas cuyo horario no incluía el trabajo nocturno $\mathrm{RR}=1,79$, (IC 95\%: 1,6-3,1).

Lie $\mathrm{Ja}^{12}$ (2006) realiza una serie de estudios casos-controles anidados en una cohorte de 44.835 enfermeras noruegas, sus resultados ponen de manifiesto un riesgo de cáncer de mama en enfermeras que han realizado horarios de trabajo nocturno durante más de 30 años $\mathrm{OR}=2,21$ (IC 95\%: 1,10-4,45) en comparación con las que no trabajaron por la noche $(\mathrm{p}=0,01)$.

S. O'leary ${ }^{13}$ (2006) estudia 576 casos de cáncer de mama y 585 controles, no encontrando una asociación significativa entre cáncer de mama y trabajos por turnos en general $(\mathrm{OR}=1,04)(\mathrm{IC} 95 \%=0,79-1,38)$, ni entre cáncer de mama y trabajos por turnos vespertinos $(\mathrm{OR}=1,08)(\mathrm{IC} 95 \%=0,81-1,44)$. Sin embargo encuentra evidencia significativa de un menor riesgo para cáncer de mama en aquellas mujeres que nunca habían trabajado por turnos (OR = 0,55) (IC 95\%: 0,32-0,94). En sus resultados el incremento de riesgo de cáncer de mama se encuentra en mujeres que tienen frecuentemente las luces de casa encendidas durante las horas de sueño (OR = 1,65; IC $95 \%=1,02-2,69)$.

Schwartzbaum ${ }^{15}$ (2007) estudia la asociación entre cáncer de mama y trabajo nocturno en una cohorte de 1.148.661 mujeres trabajadoras en Suecia. No encontrando asociación estadísticamente significativa ( $\mathrm{SIR}=0.94),(\mathrm{IC} 95 \%=0.74-1.18)$.

Stevens $\mathrm{RG}^{16}$ (2009) estudia la asociación entre problemas de visión y cáncer de mama, a través de un diseño de casos-controles en Noruega encontrando un menor riesgo de cáncer de mama en 15.000 mujeres ciegas (OR = 0,64), (I.C. 95\% = 0.21-1.49).

Este mismo autor en un estudio prospectivo, de 11.000 mujeres suecas, no encontró un riesgo significativo de cáncer de mama en mujeres con disminución moderada de la visión $(\mathrm{OR}=1.05)$, pero sus resultados ponen de manifiesto una menor riesgo de cáncer en mujeres totalmente ciegas $(\mathrm{OR}=0.47)$.

En Finlandia, analiza una cohorte de 7.396 mujeres la influencia de las horas de sueño encontrando un menor riesgo de padecer cáncer de mama sólo en mujeres con más de 9 horas de sueño (HRs: 0,28), (IC 95\% = 0,09-0,88), (p=0.03).

Una cohorte estudiada en China de 33.500 enfermeras post-menopáusicas, muestra una asociación entre la duración del sueño y el cáncer de mama, siendo sus resultados coincidentes con el estudio anterior. Las mujeres que duermen más de $8 \mathrm{~h}$ presentan un RR de 0,90 y aquellas que duermen más de 9 horas presentaron un RR de 0,81. 
Pronk A et $\mathrm{al}^{17}$ (2010), en una cohorte prospectiva de 73.049 mujeres chinas, no encontró una evidencia significativa de riesgo de cáncer de mama en mujeres que siempre trabajaron por la noche $(\mathrm{HR}=1,0)$, $(\mathrm{IC}=95 \%=0,9-1,2)$ y en mujeres con trabajo nocturno por turnos $(H R=0,99),(I C=95 \%=0,7-1,10)$. Menciona la importancia de las etnias en relación con los resultados descritos.

Pesch $\mathrm{B}^{18}$ (2010), en un estudio de 875 casos de cáncer de mama y 892 controles de trabajadoras alemanas, no encuentra un incremento significativo de riesgo de cáncer de mama en trabajadoras con turnos nocturnos frente a trabajadoras sin turnos ni trabajo nocturno $(\mathrm{OR}=0,96)$, (IC 95\% = 0,67 - 1,38). Tampoco encontró una asociación significativa al analizar el número de tunos de noche a lo largo de la vida laboral: más de 807 turnos de noche $(\mathrm{OR}=1,73)$, (IC 95\% = 0,71 - 4,22) trabajo nocturno durante más de 20 años $(\mathrm{OR}=2,48)$, (IC 95\% = 0,62 = - 9,99).

Lie JA et al $^{19}$ (2011) en 699 casos y 895 controles, encuentra un discreto exceso de riesgo para cáncer de mama en mujeres con una vida laboral de más de 30 años en hospitales $\mathrm{u}$ otras instituciones $(\mathrm{OR}=1,1)$. El trabajo de más de 12 años en horario nocturno y el acúmulo de más de 1.700 turnos durante toda la vida laboral incrementaron el riesgo $(\mathrm{OR}=1,3$ y $\mathrm{OR}=1,2)$ respectivamente.

Sus resultados evidencian una relación dosis-respuesta entre el riesgo de cáncer de mama y el número de turnos consecutivos durante más de 5 años con horarios de trabajo que incluían el trabajo nocturno: más de 4 turnos consecutivos $(\mathrm{OR}=1,4)$, más $\geq 5$ turnos consecutivos $(\mathrm{OR}=1,6)$, más de 6 turnos consecutivos $(\mathrm{OR}=1,8)$, (IC 95\% = 1,1 - 2,8).

Wu $\mathrm{AH}^{20}$ (2012), en un estudio de casos-controles anidado en una cohorte de 34.028 mujeres chinas ( 248 casos y 734 controles), no encontró relación significativa entre cáncer de mama y duración del sueño. Riesgo de cáncer de mama para más de 9 horas de sueño con respecto a menos de 6 horas $(\mathrm{OR}=0,89)$ (IC 85\%=0,64 - 1,22). Nivel de AMT6s (prediagnóstico en los casos de cáncer de mama) entre casos y controles emparejados $(\mathrm{OR}=1,00)$, (IC 905\% = 0,64 a 1,54).

Hansen $\mathrm{J}^{21}$ (2012), en un estudio casos-control (218 casos y 899 controles) realizado en Dinamarca, encuentra un exceso, no significativo, de riesgo de cáncer de mama y número años de exposición a turnos de noche (OR = 1,4) (IC 95\% = 0,9-2,1).

Davis $S^{22}$ (2012), estudia 172 casos de cáncer de mama y 151 controles, encontrando una asociación significativa entre trabajo nocturno, disminución de los metabolitos de melatonina en urinaria y aumento de las hormonas reproductoras (FSH, LH y E1C). El sueño diurno tras haber realizado un horario de trabajo nocturno evidenció una disminución de metabolitos urinarios de melatonina $\downarrow 62 \%(\mathrm{p}<0,001)$ y un aumento de hormonas reproductoras: $\mathrm{FSH}=\uparrow 62 \% ; \mathrm{LH}=\uparrow 56 \%(\mathrm{p}<0,001)$.

Durante el trabajo nocturno se evidenció una disminución de la 6-sulfatoximelatonina $=\downarrow 69 \%(\mathrm{p}<0,001)$ y un aumento de $\mathrm{FSH}=\uparrow 35 \% ; \mathrm{LH}=\uparrow 38 \%(\mathrm{p}<0,001)$.

Hansen $\mathrm{J}^{23}$ (2012) en un estudio de casos-controles anidado en una cohorte de enfermeras danesas, encontró une exceso de riesgo significativo pero variable en función del tipo de turno en trabajadoras con: turnos rotativos tras la media noche (OR $=1,8)$, (IC $95 \%=1,2-2,8)$, turno nocturno permanente, además de turnos rotatorios de noche y de día (OR = 2,9), (IC 95\% = 1,1 - 8,0), turnos rotativos a largo plazo de día y de noche (OR $=2,6$ ), (IC 95\% = 1,8 - 3,8) y turnos después de media noche en comparación con enfermeras sin fin de trabajo antes de medianoche (OR = 2,2), (IC 95\% = 1,4 - 3,2).

Menegaux F. et $a^{24}$ (2011), en el estudio de 1.232 casos de cáncer de mama y 1.317 controles en Francia, encontró que el trabajo nocturno tiene un relación con el cáncer de mama, en mujeres que inician el trabajo antes del primer embarazo a término, evidenciando un exceso de riesgo significativo en trabajadoras con trabajo nocturno durante más de 4 años antes del primer embarazo a término (OR $=1,95),(\mathrm{IC}=95 \%=1,13-3,35)$ y trabajo a turnos rotatorios que incluían la noche $(\mathrm{OR}=1,35),(\mathrm{IC}=95 \%=1,01-1,80)$. 
Knutsson et $\mathrm{al}^{25}$ (2012) en una cohorte de 4.036 mujeres, encontró un aumento de riesgo de cáncer de mama en mujeres de más de 60 años con trabajo a turnos que incluyen turno de noche. El riesgo de cáncer con turnos sin turno nocturno fue de un $\mathrm{HR}=1,23$, (IC $95 \%=0,70-2,17$ ), mientras que para los turnos que incluían el turno nocturno el valor de HR fue de $=2,02$, (IC 95\% = 1,03 - 3,95). La influencia del trabajo nocturno se evidenció también en trabajadores de menos de 60 años. Así el valor del HR para cáncer y trabajo en turnos que no implicaban nocturnidad fue de $\mathrm{HR}=1,18$, (IC 95\% = 0,67 - 2,07), mientras que para los turnos con turno nocturno fue de $H R=2,15$, (IC 95\% = 1,10 - 4,21).

Las tablas sintetizan en detalle los resultados obtenidos en relación con los objetivos formulados: estudios que analizan la asociación entre nocturnidad y cáncer de mama (tabla 7), estudios que analizan modificadores potenciales del efecto tanto intrínsecos como extrínsecos (tabla 8), estudios sobre la evidencia científica de la teoría "Chronodisruption" con la melatonina (tabla 9) y estudios que analizan factores de vulnerabilidad o población especialmente sensible (tabla 10).

Tabla 7. Estudios que analizan la asociación entre trabajo nocturno y cáncer de mama y su nivel de evidencia

\begin{tabular}{|c|c|c|c|c|c|c|}
\hline Autor & Año & Diseño & Muestra & $\begin{array}{c}\text { Medida } \\
\text { asociación }\end{array}$ & Ic $95 \%$ & $\begin{array}{c}\text { Nivel } \\
\text { evidencia }\end{array}$ \\
\hline Megdal, et al. (10) & 2005 & Meta-análisis & 13 estudios & $\mathrm{SIR}=1,44$ & $1,26-1,65$ & 1 \\
\hline Schernhammer (11) & 2006 & Cohorte prospectivo & 115.022 mujeres & $\mathrm{RR}=1,79$ & $1,6-3,1$ & 2 \\
\hline Lie JA, (12) & 2006 & Casos controles anidados cohorte & 44.835 (537 casos) & $\mathrm{OR}=2,21$ & $1,10-4,45$ & 2 \\
\hline S. O’leary, (13) & 2006 & Casos y controles & 576 casos y 585 controles & $\mathrm{OR}=1,65$ & $1,02-2,69$ & 2 \\
\hline Schwartzbaum, J. (15) & 2007 & Cohorte & $1.148 .661(6.524 \mathrm{~T} \mathrm{~N})$ & SIR $=0.94$ & $0,74-1,18$ & 2 \\
\hline \multirow{4}{*}{ Stevens RG. (16) } & \multirow{4}{*}{2009} & Casos controles & 15.000 deterioro de visión & $\mathrm{OR}=0,64$ & $0.21-1.49$ & 2 \\
\hline & & Casos controles & 11.000 disminución visión & $\mathrm{OR}=0.47$ & & 2 \\
\hline & & Cohorte prospectivo & 7.396 mujeres ( 146 casos) & HRs $=1.10$ & $0,59-2,05$ & 2 \\
\hline & & Cohorte & 33.500 post-menopáusicas & $\mathrm{RR}=1,0$ & & 2 \\
\hline Pronk A, et al. (17) & 2010 & Cohorte & 73.049 chinas ( 717 casos) & $\mathrm{HR}=0,99$ & $0,7-1,10$ & 2 \\
\hline Pesch B. (18) & 2010 & Casos y controles & 875 casos y 892 controles & $\mathrm{OR}=2,48$ & $0,62-9,99$ & 2 \\
\hline Lie JA, et al. (19) & 2011 & Casos y controles & 699 casos y 895 controles & $\mathrm{OR}=1,8$ & $1,1-2,8$ & 2 \\
\hline Wu AH. (20) & 2012 & Casos controles anidado en cohorte & 34.028 (248 casos 743 cont.) & $\mathrm{OR}=1.00$ & $0,64-1,54$ & 2 \\
\hline Hansen J. (21) & 2012 & Casos y controles & (310 casos) & $\mathrm{OR}=2,6$ & $1,8-3,8$ & 2 \\
\hline Davis S. (22) & 2012 & Casos y controles & 172 TN y 151 T de día & $(\mathrm{p}<0,001)$ & & 2 \\
\hline Hansen J. (23) & 2012 & Casos y controles & 218 casos y 899 controles & $\mathrm{OR}=1,4$ & $0,9-2,1$ & 2 \\
\hline Menegaux, F. et al. (24) & 2012 & Casos y controles & 1.232 casos y 1.317 controles & $\mathrm{OR}=1,95$ & $1,13-3,35$ & 2 \\
\hline Knutsson, et al. (25) & 2012 & Cohorte & 4.036 mujeres & $\mathrm{HR}=2,15$ & $1,10-4,21$ & 2 \\
\hline
\end{tabular}

$T=$ Turno, $T N=$ Turno nocturno 
Tabla 8. Estudios que analizan los factores potencialmente modificadores del efecto y su nivel de evidencia

\begin{tabular}{|c|c|c|c|c|c|c|}
\hline Autor & Diseño & Muestra & Potencial modificador & $\begin{array}{c}\text { Medida } \\
\text { asociación }\end{array}$ & IC $95 \%$ & $\begin{array}{c}\text { Nivel } \\
\text { evidencia }\end{array}$ \\
\hline Schernhammer ES. (11) & Cohorte prospectivo & 115.022 mujeres & $>20$ años T nocturno rotatorio & $\mathrm{RR}=1,79$ & $1,6-3,1$ & 2 \\
\hline Lie JA. (12) & $\begin{array}{l}\text { Casos controles } \\
\text { anidados en cohorte }\end{array}$ & 44.835 (537 casos) & $\begin{array}{l}\geq 30 \text { años trabajo nocturno } \\
\text { Duermen } \geq 9 \mathrm{~h}\end{array}$ & $\begin{array}{l}\mathrm{OR}=2,21 \\
\mathrm{RR}=0,81\end{array}$ & $1,10-4,45$ & 2 \\
\hline Stevens RG. (16) & 2009 & $\begin{array}{l}\text { Cohorte } \\
\text { prospectivo }\end{array}$ & 7.396 mujeres ( 146 casos) & $\mathrm{HRs}=1.10$ & $0,59-2,05$ & 2 \\
\hline Pesch B. (18) & Casos y controles & $\begin{array}{l}875 \text { casos y } 892 \\
\text { controles }\end{array}$ & $\begin{array}{l}T \text { turnos o trabajo nocturno } \\
\text { Trabajo nocturno de }>20 \text { años }\end{array}$ & $\begin{array}{l}\mathrm{OR}=0,96 \\
\mathrm{OR}=2,48\end{array}$ & $\begin{array}{l}0,67-1,38 \\
0,62-9,99 \\
\end{array}$ & 2 \\
\hline Lie JA, et al. (19) & Casos y controles & $\begin{array}{l}699 \text { casos y } 895 \\
\text { controles }\end{array}$ & $\begin{array}{l}\geq 4 \text { turnos nocturno mes } \\
\geq 5 \text { años } \geq 4 \text { turnos consecutivos } \\
\geq 5 \text { años } \geq 5 \text { turnos consecutivos } \\
\geq 5 \text { años } \geq 6 \text { turnos consecutivos }\end{array}$ & $\begin{array}{l}\mathrm{OR}=1,2 \\
\mathrm{OR}=1,4 \\
\mathrm{OR}=1,6 \\
\mathrm{OR}=1,8\end{array}$ & $1,1-2,8$ & 2 \\
\hline Hansen J. (21) & $\begin{array}{l}\text { Casos controles } \\
\text { anidados en cohorte }\end{array}$ & 310 casos & $\begin{array}{l}\text { T tarde y no nocturno } \\
\text { T rotativo después media noche } \\
\text { TN después media noche } \\
\text { T rotativos a largo plazo } \\
\text { T nocturno y rotación }\end{array}$ & $\begin{array}{l}\mathrm{OR}=0,9 \\
\mathrm{OR}=1,8 \\
\mathrm{OR}=2,2 \\
\mathrm{OR}=2,6 \\
\mathrm{OR}=2,9\end{array}$ & $\begin{array}{l}0,4-1,9 \\
1,2-2,8 \\
1,4-3,2 \\
1,8-3,8 \\
1,1-8,0\end{array}$ & 2 \\
\hline
\end{tabular}

$T=$ Turno, $T N=$ Turno nocturno

Tabla 9. Estudios que analizan la sostenibilidad de la melatonina como implicada en la "Chrono- disruption" y su nivel de evidencia

\begin{tabular}{clllll}
\hline \multicolumn{1}{c}{ Autor } & \multicolumn{1}{c}{ Diseño } & \multicolumn{1}{c}{ Muestra } & Resultados & $\begin{array}{c}\text { Medida } \\
\text { asociación }\end{array}$ & $\begin{array}{c}\text { Ic 95\% } \\
\text { evidencia }\end{array}$ \\
\hline \multirow{2}{*}{ Wu AH. (20) } & $\begin{array}{l}\text { Casos controles } \\
\text { anidados en cohorte }\end{array}$ & $\begin{array}{l}34.028(248 \text { casos y } \\
743 \text { controles })\end{array}$ & $\begin{array}{l}\downarrow \text { Melatonina y cáncer de mama } \\
\text { OR=1 }\end{array}$ & $0,64-1,54$ & 2 \\
\hline \multirow{2}{*}{ Davis S. (22) } & Casos y controles & $\begin{array}{l}172 \text { trabajo nocturno } \\
151 \text { trabajo de día }\end{array}$ & $\begin{array}{l}\text { Melatonina } \downarrow 62 \% \text { SD tras TN } \\
\text { Melatonina } \downarrow 42 \% \text { sueño N tras TN }(\mathrm{p}<0,001)\end{array}$ & 2 \\
\hline
\end{tabular}

$T=$ Turno, $T N=$ Turno nocturno, $N$ nocturno, $S D=$ sueño diurno

Tabla 10. Vulnerabilidad o población especialmente sensible

\begin{tabular}{|c|c|c|c|c|c|c|}
\hline Autor & Diseño & Muestra & Resultados & $\begin{array}{c}\text { Medida } \\
\text { asociación }\end{array}$ & Ic $95 \%$ & $\begin{array}{c}\text { Nivel } \\
\text { evidencia }\end{array}$ \\
\hline \multirow{3}{*}{ Menegaux, F. et al. (24) } & \multirow{3}{*}{ Casos y controles } & \multirow{3}{*}{$\begin{array}{l}1.232 \text { casos y } \\
1.317 \text { controles }\end{array}$} & Turno nocturno & $\mathrm{OR}=1,35$ & $1,01-1,80$ & \multirow{3}{*}{2} \\
\hline & & & Turno nocturno $\geq 4,5$ años & $\mathrm{OR}=1,40$ & $1,01-1,92$ & \\
\hline & & & TN $>4$ años antes $1^{\mathrm{er}}$ embarazo & $\mathrm{OR}=1,95$ & $1,13-3,35$ & \\
\hline \multirow{2}{*}{ Knutsson, et al. (25) } & \multirow{2}{*}{ Cohorte } & \multirow{2}{*}{4.036 mujeres } & Turnos $\sin \mathrm{TN}<60$ años & $\mathrm{HR}=1,18$ & $0,67-2,07$ & \multirow{2}{*}{2} \\
\hline & & & Turnos con $\mathrm{TN}<60$ años & $\mathrm{HR}=2,15$ & $1,10-4,21$ & \\
\hline
\end{tabular}

$T N=$ Turno nocturno

\section{DISCUSIÓN Y CONCLUSIONES}

Los resultados de la revisión sistemática realizada ponen de manifiesto la existencia de una relación entre cáncer de mama y trabajo nocturno, de los 15 estudios revisados, 14 respondían a diseños epidemiológicos longitudinales y un meta-análisis.

9 estudios evidencian una relación causal significativa entre trabajo nocturno y cáncer de mama ${ }^{10,11,12,13,19,21,22,24,25}$ y 6 estudios no llegan a resultados concluyentes ${ }^{15,16,17,18,20,23}$. 
Desde el año 2007, la IARC clasificó el trabajo nocturno como probable cancerígeno en humanos (Categoría 2A) ${ }^{1}$.

A favor de la asociación, en uno de los artículos, se ha descrito un aumento de riesgo de cáncer de mama en mujeres con turnos nocturnos durante más de cuatro años antes del primer embarazo a término (24), siendo relevante por la situación que nuestra sociedad vive actualmente, con embarazos a edades más tardías.

Los resultados ponen de manifiesto que en la interpretación de la relación cáncer de mama y trabajo nocturno debe de considerarse la experiencia laboral (número de años) en trabajo nocturno y el número de turnos nocturnos consecutivos ${ }^{11,12,21}$, evidenciándose una asociación significativa cuando son más de cinco años ${ }^{19}$.

La experiencia de exposición a trabajo nocturno y cáncer de mama previa al primer embarazo es un factor de debe considerarse también en el análisis de la relación causal ${ }^{24}$.

El aumento de riesgo de cáncer de mama podría estar en relación con la disrupción del ritmo circadiano y disminución de la síntesis de melatonina, favorecida por la exposición lumínica nocturna (Revisión de Davis 2006, relación entre la exposición nocturna a la luz y riesgo de cáncer de mama (OR=1,14) (IC95\%: 1,01-1,28) ${ }^{14}$.

En relación con lo anterior, se objetiva una menor incidencia de cáncer de mama en mujeres ciegas ${ }^{16}$

La duración del sueño, no se evidencia como factores potencialmente modificadores del efecto ${ }^{16,20}$.

Para continuar la investigación sobre la asociación cáncer de mama y trabajos en turnos nocturnos es necesario seguir realizando estudios adicionales prospectivos de larga duración y de amplio tamaño muestral, en diferentes tipos de profesiones (hasta el momento la mayoría se han realizado en enfermeras), en diferentes etnias, definiendo de forma unitaria las características de los turnos de trabajo, aumentando la significación estadística y minimizando la posibilidad de sesgos (dieta, estilo de vida, etc.). Actualmente, las investigaciones se enfocan en dos aspectos: los niveles de iluminación reales que alteran la concentración de melatonina y los tipos de trabajo que tienen mayor riesgo de desarrollar un cáncer. En espera de estos resultados se considera relevante que el esquema de rotación por turnos se ajuste a las recomendaciones ergonómicas existentes ${ }^{26}$.

Existen diferentes cuestionarios y pruebas para evaluar las diferencias en los ritmos circadianos entre los seres humanos y pruebas que identifican los efectos que los turnos pueden generar en los trabajadores ${ }^{27}$.

Una de las funciones de los Servicios de Prevención es la labor preventiva del trabajador potencialmente en riesgo, teniendo en cuenta lo descrito en la introducción en el apartado de riesgos laborales y no laborales del cáncer de mama. La legislación española contempla las posibilidades de adaptación y apoyo al trabajador especialmente sensible a riesgos presentes en su puesto de trabajo en concordancia con sus condiciones físico y psicológicas personales.

Los costes de esta patología no solamente es personal sino a nivel colectivo y social con costes directos e indirectos en relación con la incapacidad temporal que se produce ${ }^{2}$.

Recientemente en Dinamarca, primer país que considera cáncer de mama como enfermedad profesional, han recibido indemnizaciones mujeres que habían desarrollado cáncer de mama y habían trabajado en turnos nocturnos durante al menos 20 años y tenían por lo demás de bajo riesgo de cáncer ${ }^{9 ; 30}$.

Como resultado de la revisión podemos concluir la existencia de una asociación no concluyente entre el riesgo de cáncer de mama y trabajo nocturno.

La influencia como potencialmente modificadores del efecto de los siguientes factores: número de años en los que se realiza el trabajo nocturno, principalmente antes 
del primer embarazo, número de turnos nocturnos acumulados durante la vida laboral y número consecutivos de turnos nocturnos.

El aumento de riesgo de cáncer de mama en las trabajadoras con turnos nocturnos podría estar en relación con la disrupción del ritmo circadiano y disminución de la síntesis de melatonina, favorecida por la exposición lumínica durante el trabajo nocturno.

Se deben seguir realizando estudios más precisos en diferentes tipos de profesiones, etnias, etc., minimizando la posibilidad de sesgos.

\section{REFERENCIAS BIBLIOGRÁFICAS}

1. Shift-work and cancer. The IARC Monographs Vol. 98 and beyond: Exposure assessment and new results. Kurt Straif, MD MPH PhD. 2009.

2. Cáncer de Mama, Trabajo y Sociedad. Fernando Bandrés Moya y Santiago Delgado Bueno. Instituto Roche. Mayo 2012.

3. Municipal Distribution of Breast Cancer Mortality among women in Spain. Pollán M, et al. BMC Cancer 2007; 7: 78 .

4. Asociación Española contra el Cáncer. Cáncer de Mama. Incidencia; 2011.

5. Breastcancer. org. Riesgo de padecer Cáncer de Mama y factores de riesgo.

6. Occupational cancer among women: $¿$ where have we been and where are we going? Zahm SH,et al. Am J Ind Med 2003.

7. Ergonomia ocupacional sc. $\mathrm{N}^{\circ}$ 46.La rotación por turnos de trabajo probable cancerígeno para el ser humano. Vallejo González JL, 07/2009.

8. Ministerio de Empleo y Seguridad Social. INSHT. VII Encuesta Nacional de Condiciones de Trabajo 2011. 08/2012.

9. Safety Work, 10/2011.

10. Megdal, S. P., Kroenke, C. H., Laden, F., Pukkala, E. \& Schernhammer, E. S. Night work and breast cancer risk: a systematic review and meta-analysis. European journal of cancer (Oxford, England: 1990) 41, 2023-32 (2005).

11. Schernhammer, E. S., Kroenke, C. H., Laden, F. \& Hankinson, S. E. Night work and risk of breast cancer. Epidemiology (Cambridge, Mass.) 17, 108-11 (2006).

12. Lie, J.-A. S., Roessink, J. \& Kjaerheim, K. Breast cancer and night work among Norwegian nurses. Cancer causes \& control: CCC 17, 39-44 (2006).

13. O'Leary, E. S. et al. Shift work, light at night, and breast cancer on Long Island, New York. American journal of epidemiology 164, 358-66 (2006).

14. Davis, S. \& Mirick, D. K. Circadian disruption, shift work and the risk of cancer: a summary of the evidence and studies in Seattle. Cancer causes \& control: CCC 17, 539-45 (2006).

15. Schwartzbaum, J., Ahlbom, A. \& Feychting, M. Cohort study of cancer risk among male and female shift workers. Scandinavian journal of work, environment $\&$ health 33, 336-43 (2007).

16. Stevens, R. G. Light-at-night, circadian disruption and breast cancer: assessment of existing evidence. International journal of epidemiology 38, 963-70 (2009).

17. Pronk, A. et al. Night-shift work and breast cancer risk in a cohort of Chinese women. American journal of epidemiology 171, 953-9 (2010).

18. Pesch, B. et al. Night work and breast cancer - results from the German GENICA study. Scandinavian journal of work, environment \& health 36, 134-41 (2010).

19. Lie, J.-A. S. et al. Night work and breast cancer risk among Norwegian nurses: assessment by different exposure metrics. American journal of epidemiology 173, 1272-9 (2011).

20. Wu, A. H. et al. Sleep duration, spot urinary 6-sulfatoxymelatonin levels and risk of breast cancer among Chinese women in Singapore. International journal of cancer. Journal international du cancer 132, 891-6 (2013).

21. Hansen, J. \& Stevens, R. G. Case-control study of shift-work and breast cancer risk in Danish nurses: impact of shift systems. European journal of cancer (Oxford, England : 1990) 48, 1722-9 (2012). 
22. Davis, S., Mirick, D. K., Chen, C. \& Stanczyk, F. Z. Night shift work and hormone levels in women. Cancer epidemiology, biomarkers \& prevention: a publication of the American Association for Cancer Research, cosponsored by the American Society of Preventive Oncology 21, 609-18 (2012).

23. Hansen, J. \& Lassen, C. F. Nested case-control study of night shift work and breast cancer risk among women in the Danish military. Occupational and environmental medicine 69, 551-6 (2012).

24. Menegaux, F. et al. Night work and breast cancer: A population-based case-control study in France (the CECILE study). International journal of cancer. Journal international du cancer 132, 924-31 (2012).

25. Knutsson, A. et al. Breast cancer among shift workers: results of the WOLF longitudinal cohort study. Scandinavian journal of work, environment \& bealth (2012).doi:10.5271/sjweh.3323

26. Australian Council of Trade Union 2000; Scott 2002; European Foundation for the improvement and living and working condition 2000, Folkard, 2004

27. http://www.medal.org/visitor/login.aspx. Consultado el 15/12/2012.

28. Julio Domingo Jiménez Luque, Trabajo a turnos y nocturno. http://www. prevencionar.com/2011/05/10. Consultado el 23/01/2013

29. Tynes, T. et al. Incidence of breast cancer in Norwegian female radio and telegraph operators; Cancer Causes Control 1996 mar;7(2):197-204.

30. http://www.bmj.com/content/338/bmj.b1152. Consultado el 15/12/2012.

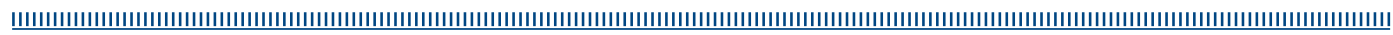

